\title{
Emphysematous pyelonephritis: A Case Report
}

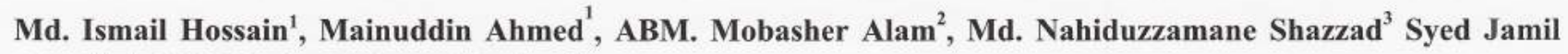 \\ Abdal $^{3}$, A K M Motiur Rahman Bhuiyan'. \\ ${ }^{1}$ OSD, Directorate General of Health Services, Deputed in BSMMU, Dhaka ${ }^{2}$ Assistant Professor, Department of Nephrology, Rangpur Medical College ${ }^{3}$ Medical Officer, Department of Rheumatology, \\ ${ }^{4}$ Associate Professor, Department of Medicine, BSMMU.
}

\begin{abstract}
:
Emphysematous pyelonephritis is an acute necrotizing infection of the renal parenchyma, prompt diagnosis and early treatment is crucial because of the high rate of mortality. We report a case of a 55 -year-old female patient with ten-year history of diabetes mellitus presented with pain in right flank, fever and malaise. She was diagnosed as a case of emphysematous pyelonephritis, and was successfully treated in our department. The case is presented along with a literature review.
\end{abstract}

Key words: Emphysematous pyelonephritis, Tc-99m DTPA (Diethylenetriamine-pentaacetic acid labeled), Nephrectomy.

[BSMMUJ 2014; 7 (1):61-64]

\section{Introduction:}

The first case of gas-forming renal infection was reported in 1898 by Kelly and MacCallum ${ }^{1}$. Since then many names have been used to describe emphysematous pyelonephritis (EPN) such as renal emphysema, pyelonephritis emphysematousa and pneumonephritis ${ }^{2}$. In 1962 Schultz and Klorfein proposed emphysematous pyelonephritis as the preferred designated name, because it stresses the relationship between acute renal infection and gas formation $^{3}$. Emphysematous pyelonephritis (EPN) is a severe, potentially fatal, necrotizing pyelonephritis with a variable clinical picture ranging from mild abdominal pain to septic shock. The majority of cases occur in diabetics with poor glycemic control while a small percentage may be due to urinary tract obstruction ${ }^{4,5}$. Previous researchers have postulated that vigorous resuscitation and appropriate medical treatment should be followed by immediate nephrectomy ${ }^{5.6}$. However, current advances in treatment, allow patients to be treated with percutaneous drainage in combination with broad spectrum antibiotics ${ }^{4,7,8}$.

We present a case of emphysematous pyelonephritis in a

Address for Correspondence: Dr. Md.Ismail Hossain, OSD, Directorate General of Health Services, Deputed in BSMMU, Dhaka, Bangladesh. email-drrussel27@gmail.com. patient with diabetes who was successfully treated with nephrectomy and antibiotics.

\section{Case report:}

A 55-years-old lady hailing from northern part of Bangladesh admitted to medicine ward through emergency department with five days history of abdominal pain, fever and vomiting. Pain was initially severe and first felt in right flank that was sudden onset and then generalized and almost continuous dull aching without any relieving and aggravating factors. The pain was associated with fever and vomiting. She was a known diabetic patient for last 10 years. On admission, she had low Glasgow Coma Scale $(12 / 15)$, fever $\left(41^{\circ} \mathrm{C}\right)$, heart rate of 100 beats per minute and a, normal blood pressure and respiratory rate. The physical examination revealed an ill-looking patient with distended abdomen. There was extremely tender right renal angle with a diffusely tender right loin. Other systems were unremarkable.

The laboratory support showed leukocytosis $(14.8 \mathrm{~K} / \mu \mathrm{L})$ with neutrophilia (88.4\%), hemoglobin $9.50 \mathrm{~g} / \mathrm{dl}$, platelet $173,010 / \mathrm{mm}^{3}$, ESR $140 \mathrm{~mm}$ in $1^{\text {st }}$ hour, creatinine 2.06 $\mathrm{mg} / \mathrm{dl}$ and random serum glucose $434 \mathrm{mg} / \mathrm{dl}$. Urinalysis revealed numerous pus cell and $\mathrm{RBC}$ but ketonuria was 
absent. The cultures from the blood and urine sample showed Escherichia coli organism.

Her Roentgenogram of KUB showed large negative shadow of air around the region of right kidney (Figure 1) which suggested emphysematous type of pyelonephritis. The sonography of the abdomen revealed the right kidney is grossly enlarged in size and shape and with total loss of cortico-medullary differentiation associated with multiple highly echogenic structure which strongly reflected as gas bubbles impacted in the renal parenchyma compatible with the diagnosis of emphysematous pyelonephritis of right kidney (Figure 2). In the following hours the patient slowly deteriorated and became hemodynamically unstable. After initial resuscitation, isotope renogram (Tc-99m DTPA) was done which showed severe obstructive uropathy in right kidney with poor functional status $(25 \%)$ (Figure 3).

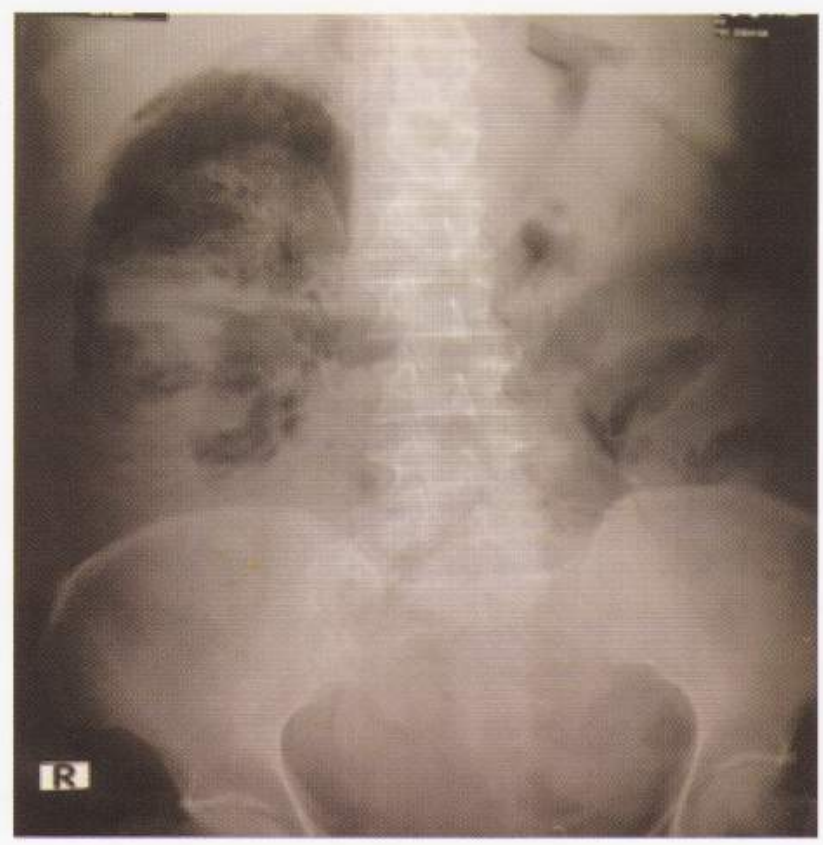

Fig-1: Plain X-ray Abdomen shows presence of air in the right renal topography

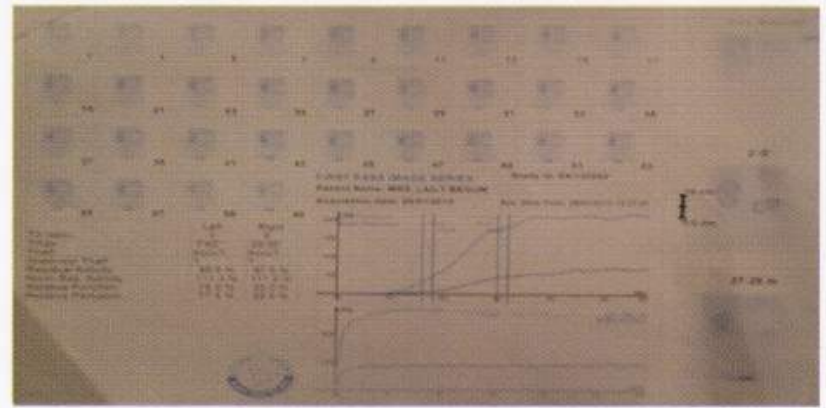

Fig-2: Tc 99m -DTPA renal scintigraphy with dynamic acquisition of sequential images revealed an increase in the time of collecting of the tracer and a delay of excretion indicating severe obstructive uropathy with poor right kidney functioning (25\%)

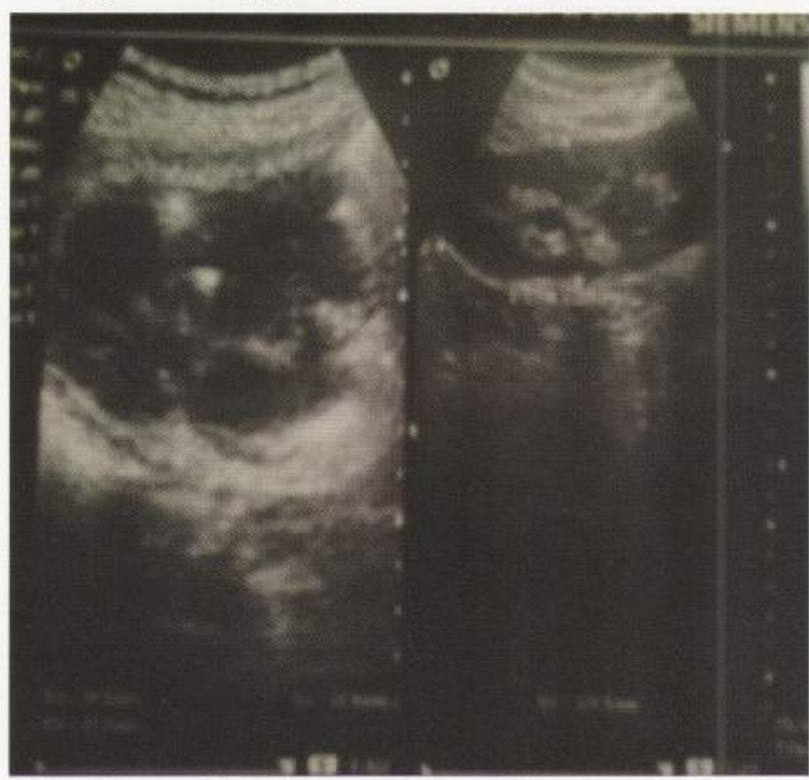

Fig-2: Ultrasonography of $K U B$ region reveals multiple hyperechoic structures within the right renal parenchyma

Ultimately, right sided nephrectomy was carried out by our surgeons and the patient was treated with broad spectrum antibiotics for 12 days. The patient had an uneventful postoperative course, and during hospital stay, her symptoms resolved completely after 16 days.

\section{Discussion:}

EPN occurs more than $90 \%$ of cases in diabetics with poor glycemic control. Other predisposing factors include urinary tract obstruction, polycystic kidneys, end stage renal disease and immunosupression ${ }^{4,5}$. Pathogenesis of EPN remains unclear, however four factors have been implicated, including gas-forming bacteria, high tissue glucose level (favoring rapid bacterial growth), impaired tissue perfusion (diabetic nephropathy) leading to further compromise of regional oxygen delivery in the kidney resulting in tissue ischemia and necrosis. Nitrogen released during tissue necrosis and a defective immune response due to impaired vascular supply. Intra-renal thrombi and renal infractions have been claimed to be the predisposing factors in non-diabetic patients ${ }^{4,5}$. 
been implicated, including gas-forming bacteria, high tissue glucose level (favoring rapid bacterial growth), impaired tissue perfusion (diabetic nephropathy) leads to further compromise of regional oxygen delivery in the kidney resulting in tissue ischemia and necrosis.

The main bacteria causing emphysematous pyelonephritis are the classical organism of urinary tract infection. The most common is Escherichia coli. Other bacteria include Klebsiella pneumoniae, Proteus mirabilis and Pseudomonas aeruginosa $a^{6,7}$. The mean patient age is 55 years old and women outnumber men probably due to their increased susceptibility to urinary tract infections. The left kidney is more frequently involved than the right one $^{4}$. Clinical manifestations of EPN appear to be similar to those encountered in classical cases of upper urinary tract infections. According to Huang and Tseng ${ }^{4}$ fever was encountered in $79 \%$ of the patients, abdominal or back pain in $71 \%$, nausea and vomiting in $17 \%$, lethargy and confusion in $19 \%$, dyspnea in $13 \%$ and shock in $29 \%$. Laboratory testing revealed elevated glycosylated hemoglobin in $72 \%$, leukocytosis in $67 \%$, thrombocytopenia in $46 \%$ and pyuria in $79 \%$. However, anaerobic infection is extremely uncommon and data appears to be a compatible with those generally reported in the literature ${ }^{8-12}$.

Various imaging techniques can be used to detect gas within the genitourinary system. Ultrasound is insensitive for the diagnosis of renal gas, but useful in diagnosing urinary tract obstruction. It is also a readily available, noninvasive method that is quite useful in the hands of experienced practitioners ${ }^{11}$. Non-contrast CT scan remains the diagnostic method of choice. In addition to showing the presence of gas, it defines the extent of the infection and can diagnose any obstruction ${ }^{4,5}$. Two staging systems, based on CT findings, have been proposed for prognostic and therapeutic reasons. Wan et $\mathrm{al}^{13}$ described two types where type-I had a mortality rate of $69 \%$ and that of type-II was $18 \%$ (Table-I). Huang and Tseng defined four classes (Table-II) ${ }^{4}$.
Table-I

Types of emphysematous pyelonephritis

Type-I: Characterized by parenchymal destruction with either absence of fluid collection or presence of streaky or mottled gas.

Type-II: Characterized as either renal or perirenal fluid collections with bubbly or loculated gas or gas in the collecting system.

\section{Table-II}

Classification of emphysematous pyelonephritis

Class 1: Gas confined to the collecting system

Class 2: Gas confined to the renal parenchyma alone

Class 3A: Perinephric extension of gas or abscess

Class 3B: Extension of gas beyond the Gerota fascia

Class 4: Bilateral EPN or EPN in a solitary kidney

The treatment of EPN remains controversial. According to some investigators ${ }^{5,6}$ vigorous resuscitation, administration of antimicrobial agents and control of blood glucose and electrolytes should be followed by immediate nephrectomy. Huang and Tseng ${ }^{4}$ proposed certain therapeutic modalities based upon their radiological classification system. For extensive EPN (classes 3 and 4) without signs of organ dysfunction antibiotic therapy combined with percutaneous catheter placement should be attempted. But nephrectomy should be promptly attempted in patients with extensive EPN and signs of organ dysfunction. Risk factors indicating poor prognosis include thrombocytopenia, acute renal failure, disturbance of consciousness and shock ${ }^{4,14}$. Falagas et $\mathrm{al}^{15}$ suggested that increased serum creatinine level, disturbance of consciousness and hypotension may need further research to confirm their potential use as risk factors for fatal outcome. Furthermore, their meta-analysis suggest that conservative treatment alone is a risk factor for adverse outcome, although one must take into consideration the different scheme, used by the authors of the studies included, when defining terms such as conservative treatment. 


\section{Conclusion:}

In high risk groups, such as diabetics, presenting with persistent upper urinary tract infection semiology that does not resolve with proper antibiotic treatment, the presence of a severe renal infection such as EPN should be considered. CT-guided percutaneous drainage or open drainage, along with antibiotic treatment, may be a reasonable alternative to nephrectomy. However, surgical intervention should not be delayed in patients with extensive disease or in those who do not substantially improve after appropriate medical treatment and drainage since the mortality rate remarkably high.

Conflict of Interest: None declaretd.

\section{References:}

1. Michaeli J, Mogle P, Perlberg S, Heiman S, Caine M.Emphysematous pyelonephritis. J Urol 1984; 131(2):203-8.

2. Schultz EH, Klorfein EH. Emphysematous pyelonephritis. J Urol 1962; 87:762-66.

3. Huang Kelly HA, MacCallum WG. Pneumaturia. JAMA 1898, 31:375-81.

4. Huang JJ, Tseng CC. Emphysematous pyelonephritis, clinicoradiological classification, management, prognosis, and pathogenesis. Arch Intern Med.2000; 160(6):797-805.

5. Shokeir AA, El-Azab M, Mohsen T, El-Diasty T. Emphysematous pyelonephritis: a 15 -year experience with 20 cases. Urology 1997;
49(3):343-46.

6. Ahlering TE, Boyd SD, Hamilton CL, Bragin SD, Chandrasoma PT, Lieskovsky G et al. Emphysematous pyelonephritis: a 5- year experience with 13 patients. J Urol 1985; 134(6):1086-88.

7. Wang JM, Lim HK, Pang KK. Emphysematous pyelonephritis. Scand J Urol Nephrol 2007; 41(3):223-29.

8. Tseng CC, Wu JJ, Wang MC, Hor LI, Ko YH, Huang JJ. Host and bacterial virulence factors predisposing to emphysematous pyelonephritis. Am J Kidney Dis 2005; 46(3):432-39.

9. Christensen J, Bistrup C. Case report: emphysematous pyelonephritis caused by clostridium septicum and complicated by a mycotic aneurysm. Br J Radiol 1993; 66(789):842-43.

10. Mallet M, Knockaert DC, Oyen RH, Van Poppel HP. Emphysematous pyelonephritis: no longer a surgical disease? Eur J Emerg Med 2002; 9(3):266-69.

11. Stone SC, Mallon WK, Childs JM, Docherty SD. Emphysematous pyelonephritis: clues to rapid diagnosis in the Emergency Department. J Emerg Med 2005; 28(3):315-19.

12. Vetere NS, Monti J, Gutman D. A case report of emphysematous pyelonephritis secondary to ureteral obstruction in a nondiabetic patient. Am J Emerg Med 2006; 24(6):749-50.

13. Wan YL, Lee TY, Bullard MJ, Tsai CC. Acute gas-producing bacterial renal infection: correlation between imaging findings and clinical outcome. Radiology 1996; 198(2):433-38.

14. Wan YL, Lo SK, Bullard MJ, Chang PL, Lee TY. Predictors of outcome in emphysematous pyelonephritis. J Urol 1998;159(2):369-73.

15. Falagas ME, Alexiou VG, Giannopoulou KP, Siempos II. Risk factors for mortality in patients with emphysematous pyelonephritis: J Urol 1968; 98:742-46. 